Rashid G. Alakbarov ${ }^{1}$, Ogtay R.Alakbarov ${ }^{2}$

DOI: 10.25045/jpit.v08.i1.05

Institute of Information Technology of ANAS, Baku, Azerbaijan

1ashid@iit.ab.az, ${ }^{2}$ oqtayalakbarov@yahoo.com

\title{
MOBILE CLOUDS COMPUTING: CURRENT STATE, ARCHITECTURE AND PROBLEMS
}

The article analyzes the current state of devices and their distribution dynamics on different indicators. Development dynamics of mobile cloud technologies is researched. Advantages of these technologies and emerging challenges by using these technologies are analyzed. Meanwhile, provision of demand to computing and memory resources of mobile devices challenges by using these technologies are researched.

Keywords: mobile computing clouds, mobile devices, computing and memory resources, cloudlet, computing clouds, mobile trade mobile, healthcare, cloud services.

\section{Introduction}

At present, studies on the efficient use of computing and memory resources in data processing centers with the help of Cloud Computing (CC) have been intensively conducted in the world. Such systems, which have big computing and memory resources, are established on the basis of computer networks with high-speed communication channel. CC technology enables the organizations to use of computing and memory resources of processing centers more efficiently. With the help of this technology, a user's data is processed and stored on cloud servers, and at the same time, the results can also be reviewed with the help of browsers [1,2]. CC service provides the use of a wide range of clustering and virtualization of computing and memory resources of processing centers. Thus, the CC system is an Internet service composed of hardware and software, which ensures the use of remote computer resources (computing and memory resources, software, and data etc.) through an appropriate web interface for the Internet users.

$\mathrm{CC}$ technology allows users to access powerful computing and memory resources, and the user is not interested in where these resources are located and installed. With the help of these services, the efficient use of computing and memory resources in data processing centers is granted. Hence, the time to resolve the problem is reduced, and the network is loaded less [3]. By optimal distribution of memory resources available in processing center among the users, cloud technology attracts more users to the system and creates an opportunity to promptly achieve results. In recent years, mobile users have started to widely use the services of CC technology.

Rapidly increasing use of mobile devices (laptops, tablets, smartphones, etc.) in the world and their connection to computing clouds over the internet with the help of certain telecommunications technologies (GPS, 3G, 4G, Wi-Fi etc.) have enhanced the development of new technology - Mobile Cloud Computing. This paper reviews more efficient use of computing and memory resources of mobile computing clouds (MCC), which are widely used recently. Obviously, capabilities (computing and memory resources) of any mobile device are limited. However, the users use these devices to solve problems that require large computing and memory resources. Cloud computing technology is widely used, in this regard. Thus, the problem of mobile users related to the insufficient computing and memory resources can be resolved through the use of cloud technologies.

\section{Analysis of the current status of mobile devices}

The main purpose of MCC is to provide convenient and fast use of computing clouds services to the mobile users. Mobile services have become widespread with the advent of CC technology. These devices are playing a very important role in our life in terms of entertainment and communication, and at the same time, they do not have time and space restrictions.

$\mathrm{CC}$ technology has been used widely in recent years since it offers virtual computing and memory resources, platform and software, using cheaper server and network resources. Cloud 
computing, which is easy to use with lower price, creates great opportunities for a wide use of mobile applications in mobile environment, and can provide various types of services to mobile users.

Users, on the one hand, demand high computing resources, and wish to use mobile devices, which are small in size, on the other hand. Currently, cloud technologies are used to eliminate the mentioned problem of mobile devices (lack of computing and memory resources). MCC is a new platform, which is a combination of computing clouds and mobile devices, and ensures the mobile users to solve complex issues and to store large data sets in the clouds [4]. The technical capabilities of mobile devices (computing and memory resources) are limited. To overcome this restriction cloud technologies are widely used. Using the services of computing clouds, mobile users are able to resolve any problem. In recent years, lower prices of cloud servers allow mobile users to benefit from a wide use of these services. Currently, many companies have developed numerous software applications (Google, Gmail, Maps and Navigation Systems for Mobile, Voice Search, Mobile Me from Apple, Live Mesh from Microsoft) for mobile device users, which have lead them to extensive use of MCC [5]. This article examines the current state of development dynamics of mobile devices by various indicators. The production of mobile devices and the development dynamics of their users are shown in Table1.

\begin{tabular}{|l|c|c|c|c|c|}
\hline & 2014 & 2015 & 2016 & 2017 & 2018 \\
\hline $\begin{array}{l}\text { The number of mobile } \\
\text { users (billions) }\end{array}$ & 5,674 & 5,808 & 5,945 & 6,085 & 6,228 \\
\hline $\begin{array}{l}\text { The number of mobile } \\
\text { device (billions) }\end{array}$ & 7,733 & 8,627 & 9,628 & 10,825 & 12,165 \\
\hline $\begin{array}{l}\text { The number of mobile } \\
\text { devices per user } \\
\text { (billions) }\end{array}$ & 1,36 & 1,49 & 1,62 & 1,78 & 1,95 \\
\hline
\end{tabular}

Table1. Development dynamics of mobile users and devices

As shown in above table, the number of mobile devices to be produced in the world in 2018 will be 12.165 billion, while the number of mobile users will be 6.228 billion. This will lead to the rapid increase in the number of MCC users over the next few years.

The development dynamics of the Internet users in the world is shown in Figure 1. As shown in the chart, the number of personal computers (desktop) connected to the Internet is declining after 2016. Whereas, the number of mobile devices with high-speed Internet access is likely to be increased. In 2016, the number of mobile Internet users will reach 2.16billion.

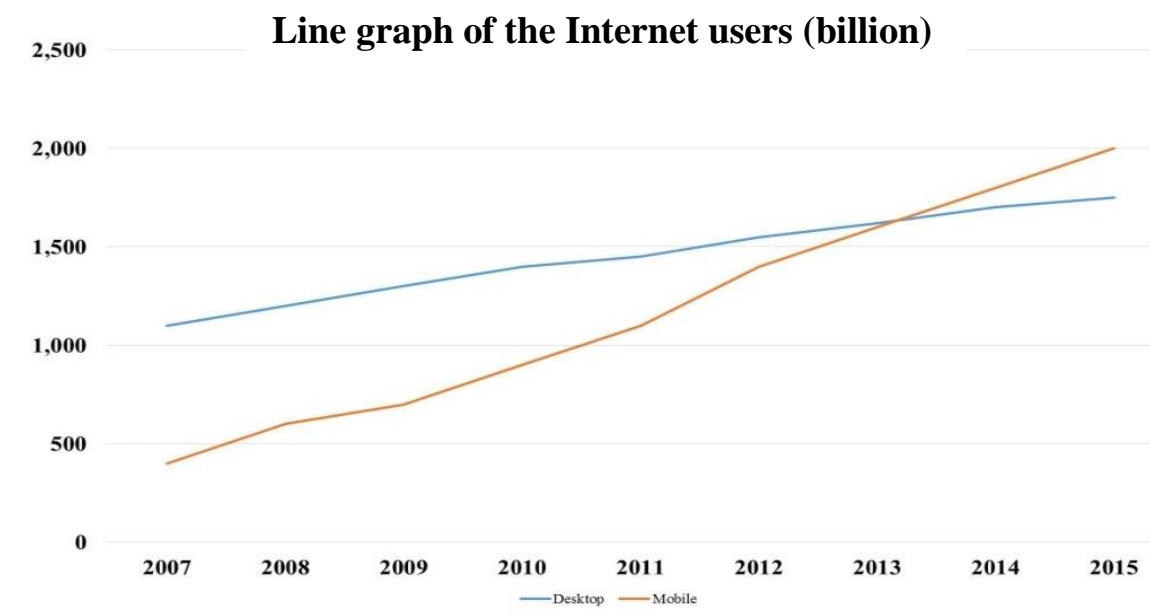

Figure 1. Development dynamics of the Internet users 
Recently, the number of applications developed for mobile devices has started to rapidly increase (Figure 2). This, in turn, causes overload of the network due to the intensive use of these applications. In 2017, the number of applications created for mobile devices will approach 300 million.

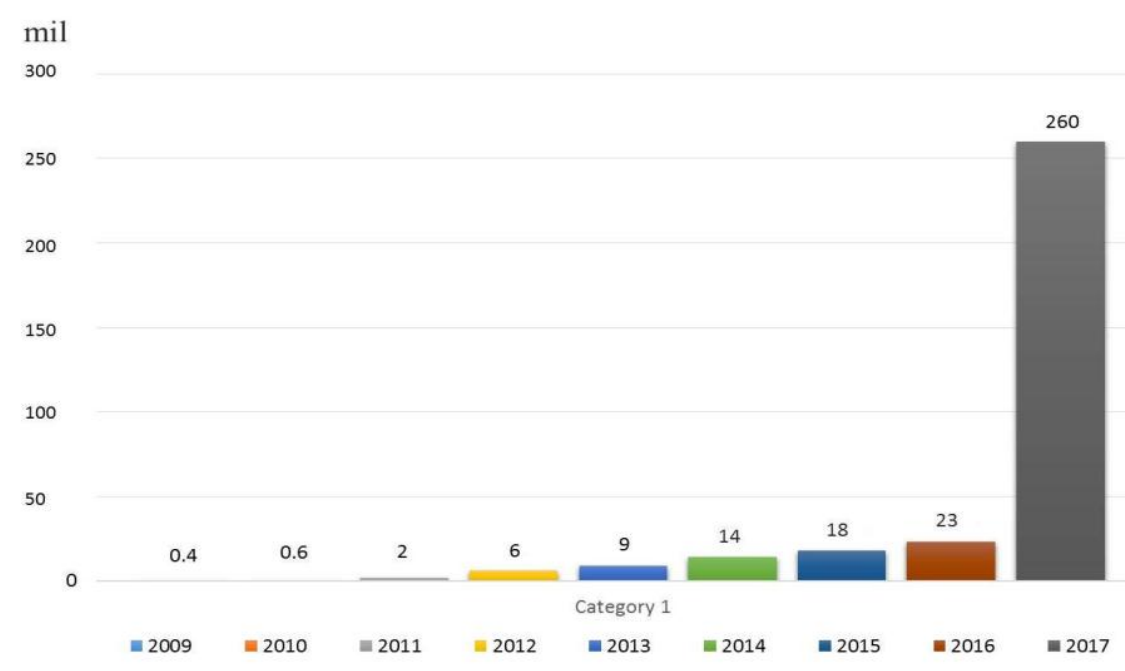

Figure 2. Dynamics of Mobile Application Development

The dynamics of communication channel load of smartphones when using the Internet within a month is shown in Figure 4. As it is seen from the figure, a monthly traffic load in 2019 is likely to reach 18.24 Ebyte (1018 bytes). This will result in network loading, delays to access data and results on time.

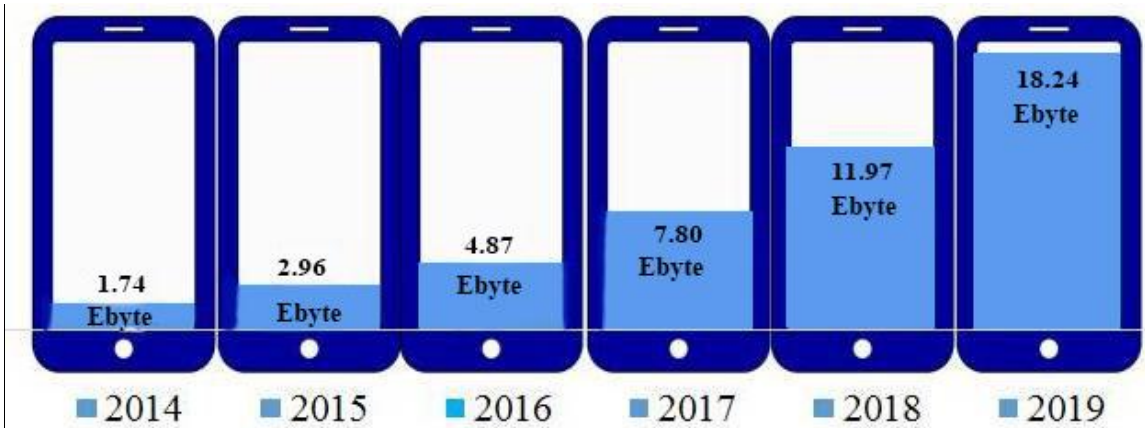

Figure 3. Monthly Internet traffic of smartphones worldwide (Exabyte)

Distribution of mobile devices among the manufacturing companies is shown in Figure 5. As shown in the pie chart, the production of mobile devices is distributed among three companies across the world, namely among Samsung (30\%), Apple (26\%), Huawei (21\%).

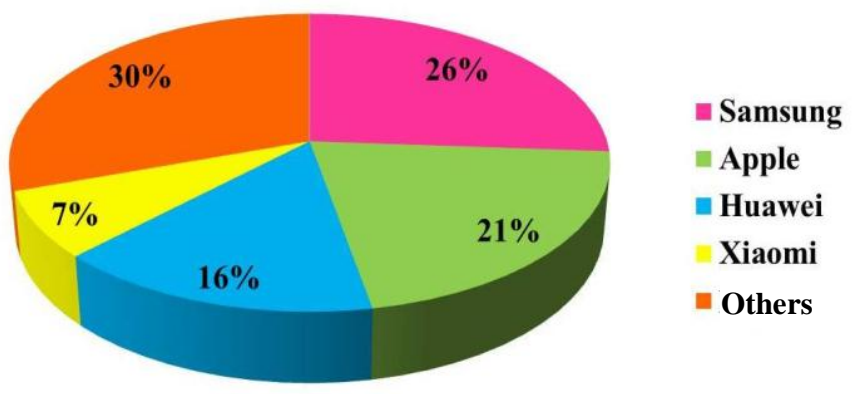

Figure 4. Distribution of mobile device manufacturer

Distribution of operating systems used in mobile devices is shown in Figure 6. As shown in 
the pie chart, mobile devices are using Google's Android (85.2\%) and Apple's iOS (13.8\%) operating systems.

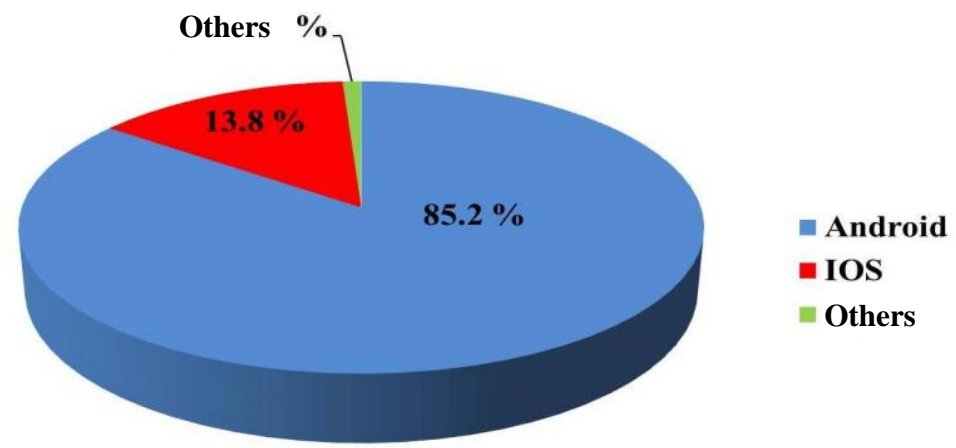

Figure 5. Distribution of operating systems used in mobile devices

According to Gartner analysts, the number of the mobile Internet users in 2016 was 2.16 billion. In 2018, this figure will rise to 2.56 billion. Thus, the rapid increase in the number of mobile Internet users seems to be observed during the following 2-3 years. This, in turn, provides an opportunity to network load and results in inefficient use of these applications, as well as causing delays in delivering the processed data to the user. MCC is used to coupe with the above-mentioned problems.

\section{MCC architecture and application fields}

Data processing and storage in MCC is implemented beyond the mobile devices. In MCC, mobile devices are connected to cloud computing system over the Internet with the help of the base stations (GPS, 3G, 4G, Wi-Fi etc.) and use required services. Currently, users are widely using three types of cloud services (IaaS, PaaS and SaaS) (Figure 6). IaaS (eng. Infrastructure as a Service) provides the users to use computing and memory resources of cloud systems (e.g., Amazon Elastic Computing Cloud - EC2 and Amazon S3 - Simple Storage Service).

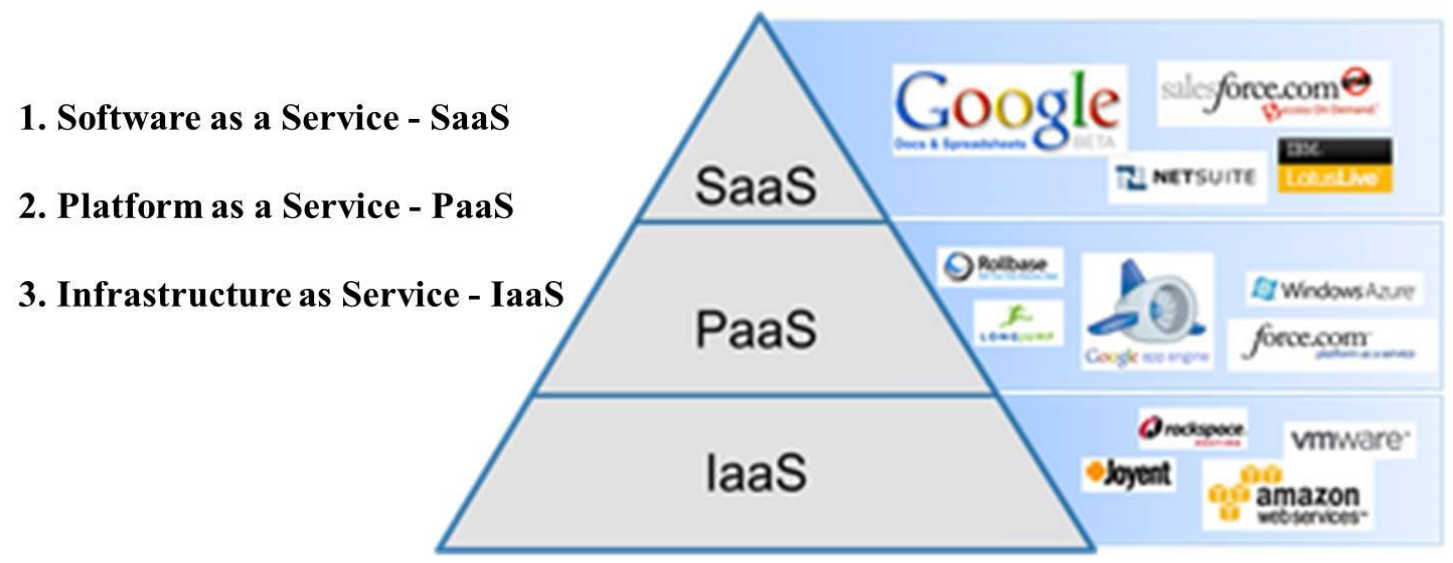

Figure 6. The services used in MCC

PaaS (Platform as a Service) enables the service user to use operating systems and custom software applications (Google App Engine, Microsoft Azure, My SQL etc.) on virtual servers. SaaS (Software as a Service) enables the service users to solve their problems using the applications (Google Apps, Google Docs, Autodesk etc.) and software located on clouds computing servers. With SaaS service, the user can get the results enabling the necessary software on clouds servers via the Internet without downloading the resident part of the program to his/her computer. The applications run on the servers of SaaS provider and send the processed data to the user. Thus, user does not purchase the application and only pays for what he uses.

Currently, although the centralized clouds used by the users have high computing and memory resources, it is not capable to provide high speed deliver of processed data to the users. 
The rapid growth of the number of mobile users in clouds causes the network loading, which also leads to delays in the delivery of processed data to the user. To overcome the mentioned shortage, the resources of these clouds should be placed closer to the user.

When using cloud services the user uses two modes, namely offline and online. For example, a user accesses to virtual computing machine on clouds for the problems that require high computing power. He sends the problems to clouds and gets a result in a while. In this mode, there is no direct connection between the user's computer and the cloud during the time needed to resolve the problem. Nevertheless, there are such issues that there is a connection between the user and the clouds for their solution until the completion of the process. And this leads to the network loads in the resolution of the mentioned issues. To eliminate the network loads, MCC technologies are used. It is often called decentralized cloud technologies. MCC's architecture is shown in Figure 7. As it is seen from the figure, the system consists of several components: mobile users (mobile devices, smartphones etc.), mobile network operators, wireless connection devices (Wi-Fi Access Point), Internet service providers (ISP), cloud computing providers (Amazon, IBM Google, Microsoft, etc.). As the scheme displays, mobile users are connected to CC system over the Internet with the help of base stations (mobile communication, Wi-Fi Access Point) and use its computing and memory resources. More network loading and delays occur in processed data delivery during these type connections. At the same time, the prices are high. Mobile CC systems with hierarchical structure are used to eliminate the above-mentioned shortcomings [4.7].

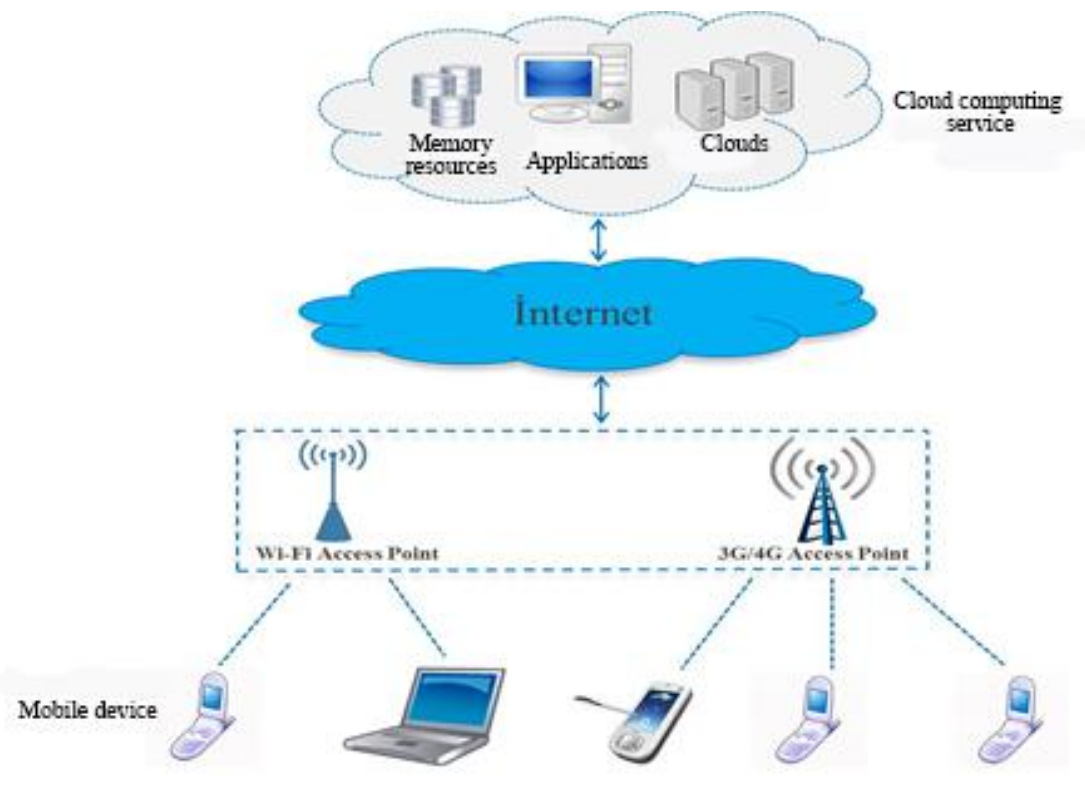

Figure 7. The architecture of MCC

The research shows that the volume of mobile device production and the number of mobile Internet users is rapidly growing. The mobile internet users are predicted to be 6.5 billion by 2020 . Mobile CC systems with such level architecture are not able to provide high quality services to the users. In other words, network loading, delayed delivery of results to the users, high costs of service, occurrence of delays, traffic loadings and other occurrences in these systems do not provide high quality service. To overcome these problems, mobile CC systems with hierarchical structure (2 level) are used (Figure 8).

As seen from the scheme, mobile Internet users download the needed applications to closer cloudlet system servers and use them, which, in turn, allow avoiding the Internet loading. This architecture, at least, partially improves the performance of some parameters. High level CC system servers are located in the hierarchical structured architecture, and the Cloudlets are located close to the base stations in the $2^{\text {nd }}$ level. The questions of defining to place Cloudlets near to which base stations and what characteristics of they have are of urgent issues. 


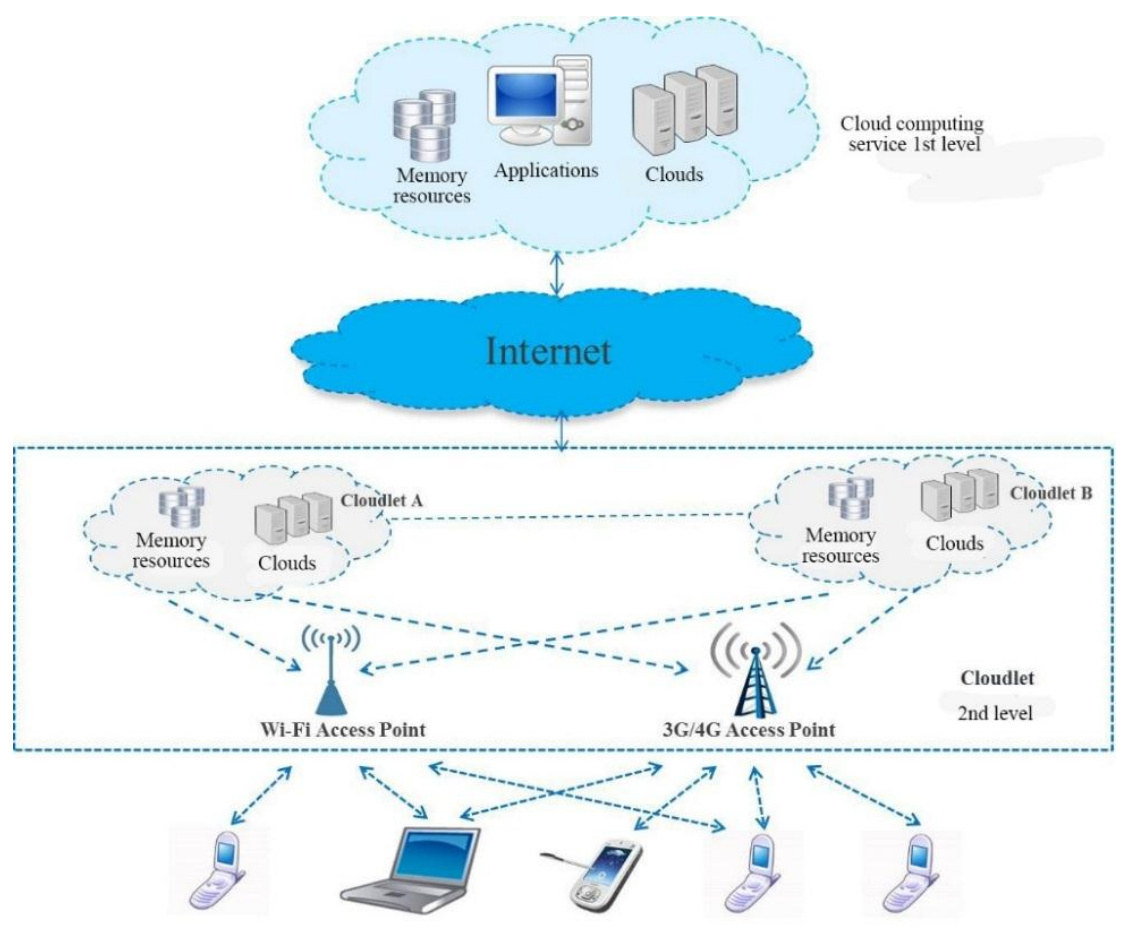

Figure 8. MMC architecture with the hierarchical structure

Thus, for wider use of cloud technologies in mobile devices, mobile servers (Cloudlet) are built near the base stations of mobile operators. Cloudlet (small clouds) is a device (server) placed close to users, which provide smore rapid delivery of necessary information from the central servers to the user. When the user finishes his work, if necessary, the data is loaded to the central servers again. Cloud services necessary for users are carried out through Cloudlet servers [7-9]. For instance, if a user needs to work in any application (SaaS service), calls the application to the mobile server closer to him, and works with the application within the necessary period of time and then sends it to the central server. This reduces the cost of cloud service, and the time spent for the problem solution is reduced and the network is loaded less. On the other hand, the demand for computing and memory resources can be met by using the traditional centralized cloud services. However, the delays occur in the acquisition of results or data in this type of cloud services. For example, new online translation service has been developed by Google (Google Translate API), with the help of which the users speaking different languages to communicate with one another over the Internet. The mobile devices (smartphones) used service do not perform the translation per se, and they send the words and sentences to Google Cloud Platform servers. Translation is performed on the server, and the results are sent to people speaking to one other. This type of service runs slowly (with delays). Delays occur in its delivery over the network, while the translation is usually performed within a few seconds. Therefore, it would be better to place translation program on the local servers (Cloudlets) close to users and creating opportunity for prompt real-time translation. At the same time, when using SaaS service, placing the application necessary for users on closer cloud servers reduces the service cost, thus increases its speed and quality.

Currently, benefitting from the services of mobile cloud providers, millions of mobile users around the world are widely using mobile applications (in mobile commerce, mobile education, mobile health, mobile games etc.) $[4,6,10]$.

Mobile commerce: carrying out trade through mobile devices. Mobile users perform mobile banking, mobile brokerage operations, mobile marketing and advertising through mobile commerce. Mobile commerce applications perform a number of operations that require mobility functions, such as mobile transactions and payments, mobile messaging and mobile booking. Since mobile commerce has a variety of products and applications, the problems as poor network coverage, various and very complex configuration of mobile devices, as well as security and privacy arise. To 
resolve the mentioned problems, it is desirable to transfer the mobile commerce applications to cloud environment. For some security issues, public key infrastructure (PKI) is used.

Mobile Health: medical applications in the mobile environment are called mobile medical applications and used for medical treatment, patient monitoring, prompt diagnoses and so on. Mobile health care provides the followings:

- medical monitoring services for the observation of the patient via the Internet at any place and any time;

- development of emergency management system to ensure the effective and timely availability and control of ambulance in the case of any emergency, event or accident;

- organization of alarm system installed medical mobile devices for measuring pulse, blood pressure and the level of alcohol in the emergency situations;

- storing patients' health data used in medical practice and research.

Storing mobile medical applications in clouds provides the users with easy and fast access to resources anytime and anywhere. Nevertheless, security solutions must be offered in order to protect the confidentiality of patients' health data in clouds.

Mobile Learning is the training through mobile devices. This structure is based on e-learning and mobility. The services if the traditional mobile learning applications over the network are expensive and have some limitations due to the poor network speed and limited teaching resources. The cloud-based mobile learning applications have been proposed to solve these problems. For example, with the help of powerful processing capabilities and large memory capacity of clouds, the users are provided with high-quality training programs, faster processing and more long-term energy.

Mobile games: due to the limited processing capabilities of mobile devices, they are mainly using simple games. The cloud technologies are used to take advantage of more complex and attractive games. A part of the game application that requires large computing resources are placed in clouds and users are able to control the game through the interface located in their mobile devices. This, in turn, gives a lot of advantages such as reducing power consumption, and faster use of game applications due to processing power of cloud.

Developed mobile software applications do not depend on the operating systems mobile devices and the type of device. Therefore, the number of mobile users who use the cloud service is rapidly growing day by day. In this case, the mobile devices with minimal computing and memory resources act as a thin client terminal connected to the Internet.

The advantages of MCC include $[7 \div 9]$ :

- increases the working period of batteries of mobile devices;

- increases the capacity of computing and memory resources of mobile devices;

- increases the reliability by storing user data in a few reserve computers and this, in turn, reduces the risk of data loss in mobile devices;

- dynamic distribution of resources - it ensures the user's access to the resources anytime without ordering them in advance;

- increases the transmission capacity of the network;

- cost-efficiency of services;

- provides access to the user data anywhere around the world by organizing their storage in clouds;

- increases scalability. the system provides user access to computing and memory resources in a short period of time due to the flexible resource capabilities;

- security capabilities of the system. Mobile users faced with the problems related to data storage on cloud servers and their protection when they use cloud services. Though the service providers protect their users' data through various security applications, at the same time, they have the opportunity to review this data. Therefore, the problem of confidentiality is considered to be very important. For the solution of such problems and for the prevention of illegal interference, several security programs written for mobile devices are used. These 
security programs tend to use a certain part of computing and memory resources of mobile devices. Therefore, it is advisable to transfer the threat detection programs from mobile devices to cloud servers. In this regard, Cloud AV platform has been developed; it provides numerous services to detect malicious programs both on clouds and mobile devices;

- easy integration. The users' demand can be satisfied by integrating the services of various providers with each other via the Internet and cloud.

The extent of the volume of technical capabilities (virtual machines, number, memory size, network transmission capabilities, etc.) of servers used in the creation of the small clouds close to base stations is of great importance. At the same time, the provision of the link among the small clouds is also an urgent issue.

\section{MCC problems and their solutions}

The main problems of MCC arise due to the features of mobile applications and wireless network, as well as the limited opportunities of their resources. These problems complicate the development of the applications and their functioning in mobile and distributed devices. In mobile cloud computing environment, the limitations of the technical capabilities of mobile devices, the quality of wireless communication, and the diversity of applications are of important factors affecting the measurement of clouds.

Limitations of mobile devices. Reviewing the mobile devices in cloud environment, restrictions of resources must be considered first. Although many settings of mobile devices as the processor and memory resources, screen size, wireless communication, sensor technology and operating systems have been significantly improved, there are still serious problems related to the use of energy sources and the complex applications. Smartphones using Android and Windows Mobile operating systems are left behind PC's processing capacity by 3 times, RAM capacity - 8 times, and files storage - 5-10 times [10].

Communication quality. Unlike wired networks using physical connection, which provides fixed coverage, data transmission is constantly changing in mobile cloud computing environment. In addition, data center of large companies and the resources in the Internet service provider are often located very far from end users, especially the users of mobile devices, and it causes problems in obtaining results rapidly. Network delays in wireless network may last $200 \mathrm{~ms}$, and only $50 \mathrm{~ms}$ in traditional wired networks.

Division of applications. Due to the problem of the limited resources of mobile devices, they can not use the applications that require large computing and memory resources or such applications require high energy consumption when they run. Therefore, the applications are divided into several parts and use MCC's resources. The main calculation part of the applications is processed by the cloud, while mobile devices solve only some simple problems.

Thus, the key indicators affecting effective use of data processing in MCC include data processing time in data center and mobile device, network delays and data delivery time. The following strategies can be used to solve the mentioned problems:

- to place Cloudlets close to the user to reduce the delay time in MCC;

- to place and optimize main and auxiliary parts of the applications in the cloud and mobile devices;

- to build Cloudlet-based network infrastructure;

- to cluster the Cloudlets services used there (IaaS, PaaS, SaaS, etc.);

- to use mobile cloud computing systems with hierarchical architecture;

- to identify technical capacity of computing and memory resources of Cloudlets.

When using cloud technology the users base on the following criteria:

- expense required to solve the problem;

- time to solve the problem;

- ensuring the security of users' data; 
- fast and reliable delivery of data and results to the users.

The specified criteria depend on how far the cloud servers from the user in terms of geographic location, capacity of the virtual computing machine and the loading rate of the network. Therefore, they are trying to locate the servers of mobile cloud technologies close to the users. It is preferable to set up the Cloudlets near to each base station. Nevertheless, such mobile CC systems are expensive and at the same time, the system is not effectively used. Therefore, any areas of Cloudlets deployment (near educational institutions, shopping centers, leisure areas etc.) should be monitored and analyzed. Furthermore, the most used services in the determined zones should be defined. Then, the technical capabilities (computing and memory resources) of the Cloudlet system to be set in the same zone must be identified. The areas, where a group of people gather in large numbers can be identified and after analyzing a large volume of data they use, the location of Cloudlets in these zones can be decided.

\section{Conclusion}

The article analyzed the dynamics of the current situation and development of mobile devices. MCC's architecture and its application areas were examined, and the advantages of the hierarchical structure of the system were presented. The problems arisen from the use of MCC and their possible solutions were explored. The conditions necessary for the deployment of cloudlets in MCC were investigated.

This research was carried out with the financial supported of the Science Development Fund under the President of the Republic of Azerbaijan - Grant No EIF-2014-9 (24) KETPL-14/02/1.

\section{References}

1. Marios D. Dikaiakos, G. Pallis, D. Katsaros, P. Mehra, A. Vakali. Cloud Computing Distributed Internet Computing for IT and Scientific Research // IEEE Internet Computing, 2009, № 9, pp.10-13.

2. Alguliyev R.M., Alekperov R.K. Cloud Computing: Modern State, Problems and Prospects // Telecommunications and Radio Engineering, 2013, vol.72, no.3, pp.255-266.

3. Alakbarov R., Pashayev F., Hashimov M. A Model of Computational Resources Distribution Among Data Center Users // International Journal of Advancements in Computing Technology, 2015, vol.7, no.2, pp.1-7.

4. Dinh H.T., Lee C., Niyato D., and Wang P. A survey of mobile cloud computing: Architecture, applications, and approaches // Wireless Communications and Mobile Computing, 2013, vol.13, no.18, pp.1587-1611.

5. Qi H., Gani A. Research on Mobile Cloud Computing: Review, Trend and Perspectives. https://arxiv.org/ftp/arxiv/papers/1206/1206.1118.pdf.

6. Goyal M., Singh S. Mobile Cloud Computing // International Journal of Enhanced Research in Science Technology \& Engineering, 2014, vol.3, no.4, pp.517-521.

7. Liu F., Shu P., Jin H., Ding L., Yu J., Niu D., Li B. Gearing resource-poor mobile devices with powerful clouds: architectures, challenges, and applications // IEEE Wireless Communication, 2013, vol.20, no.3, pp.14-22.

8. Satyanarayanan M., Bahl P., Caceres R., Davies N. The case for vm-based cloudlets in mobile computing // IEEE Pervasive Computing, 2009, vol.8, no.4, pp.14-23.

9. Li Y., Wang W. The Unheralded Power of Cloudlet Computing in the Vicinity of Mobile Devices / Globecom 2013 - Wireless Networking Symposium, pp.4959-4964.

10. Kumar L. (16084), Nishant Malik (16104), GouravAgghi (16067), Ajay Anand Mobile Cloud Computing (16951). IJRIT International Journal of Research in Information Technology, 2014, vol.2, no.9, pp.787-792. 